\title{
Competência oocitária: importância e fatores determinantes in vivo e in vitro
}

\author{
[Oocyte competence: importance and determinant factors in vivo and in vitro]
}

\section{"Revisão/Review"}

\section{Letícia Ferrari Crocomo $^{1 *}$, Fernanda da Cruz Landim-Alvarenga ${ }^{2}$, Sony Dimas Bicudo ${ }^{2}$}

\author{
${ }^{1}$ Instituto de Ciências Agrárias, Universidade Federal de Minas Gerais (ICA-UFMG), Montes Claros - MG, Brasil. \\ ${ }^{2}$ Faculdade de Medicina Veterinária e Zootecnia, Universidade Estadual Paulista (FMVZ-UNESP), Botucatu-SP, Brasil. \\ *Autor para correspondência/Corresponding author: E-mail: leticia.crocomo@gmail.com
}

\begin{abstract}
Resumo
As biotécnicas reprodutivas impulsionaram a pecuária no cenário do agronegócio mundial em virtude da redução do intervalo entre gerações, acelerado ganho genético e intensificação da produtividade. Nesse âmbito, existem, no entanto, desafios biológicos e fisiológicos que, apesar do avanço científico e biotecnológico, ainda não foram solucionados. A superovulação associada à aspiração folicular e a produção in vitro de embriões a partir de complexos cumulus-oócitos (COCs) que, outrora, entrariam em atresia, tem sua eficiência limitada, basicamente, pela qualidade oocitária, a qual está diretamente relacionada à competência do oócito em completar sua capacitação, representada pela maturação nuclear e citoplasmática, além de suportar a fertilização e embriogênese inicial. Esse processo de capacitação oocitária, tanto in vivo quanto in vitro, determina, portanto, o potencial de desenvolvimento embrionário e envolve uma complexa interação de moléculas regulatórias além de alterações moleculares e estruturais, os quais estão, ainda, sob a influência de fatores intra e extra-ovarianos. Sendo assim, dada a importância do oócito para fertilidade da fêmea, a presente revisão tem como intuito discutir os principais aspectos implicados no contexto da capacitação oocitária e seus fatores interferentes assim como apresentar possíveis métodos de seleção de COCs competentes.
\end{abstract}

Palavras-chave: oócito; cumulus; capacitação; competência; biotécnicas reprodutivas.

\begin{abstract}
Reproductive biotechnology promoted beef cattle production growth in the global agribusiness scenario due to the reduction of generation interval, accelerated genetic gain and productivity enhancement. In this context, however, there are biological and physiological challenges that, despite scientific and biotechnological advances, have not yet been overcome. Superovulation associated with follicular aspiration and in vitro production of embryos from cumulus-oocyte complexes (COCs) that would ultimately develop atresia, have its efficiency limited, basically, by oocyte quality, which is directly related to oocyte competence to complete its capacitation, represented by nuclear and cytoplasmic maturation, as well as supporting the fertilization and initial embryogenesis. This process of oocyte capacitation, both in vivo and in vitro determines, therefore, the embryo development potential and involves a complex interaction of regulatory molecules in addition to molecular and structural changes, which are still under the influence of intra and extra-ovarian factors. Thus, given the importance of the oocyte for female fertility, the present review aims to discuss the main aspects involved in oocyte capacitation, its interfering factors, and to present possible selection methods of competent COCs.
\end{abstract}

Keywords: oocyte; cumulus; capacitation; competency; reproductive biotechnology.

\section{Introdução}

O melhor aproveitamento de matrizes e reprodutores aliado à obtenção de maior número de descendentes, redução do intervalo de geração e acelerado ganho genético impulsionou a intensificação e aplicação comercial de biotécnicas reprodutivas envolvendo a superovulação, aspiração folicular, produção in vitro de embriões (PIV), criopreservação de gametas e transferência embrionária nas mais variadas espécies animais. $\mathrm{O}$ aprimoramento tecnológico revolucionou, 
portanto, a pecuária a nível mundial pela garantia de produtividade mesmo diante de obstáculos reprodutivos, sanitários e ambientais (Choudhary et al., 2016). A possibilidade de desenvolvimento embrionário a partir de oócitos recuperados de folículos que outrora entrariam em atresia consiste, no entanto, num desafio biológico e biotecnológico (Merton et al., 2003). Além disso, apesar dos recentes avanços científicos observados na PIV, em especial de bovinos, relacionados, principalmente, à composição do meio de cultivo e condições laboratoriais in vitro, apenas um terço dos oócitos aspirados alcança o estágio de blastocisto e muitos destes embriões apresentam baixa viabilidade comparada àqueles obtidos in vivo (Abd El-Aziz et al., 2016).

Esta baixa eficiência está diretamente relacionada à competência oocitária em suportar a fertilização e embriogênese inicial, a qual é adquirida ao longo da oogênese envolvendo interação com as células da granulosa ao seu redor e progressão, de maneira sinérgica, dos eventos bioquímicos, moleculares e estruturais que caracterizam a maturação nuclear e citoplasmática do oócito (Sirard et al., 2006). Além disso, não apenas a embriogênese inicial, mas a implantação do embrião no endométrio, manutenção da gestação, crescimento fetal e até mesmo algumas enfermidades no animal adulto também estão diretamente relacionados à qualidade oocitária uma vez que o oócito confere suporte, em termos molecular e bioquímico, aos estádios posteriores do desenvolvimento. Deste modo, apesar da sua importância para fertilidade da fêmea, devido as suas peculiaridades, o oócito é altamente sensível à fatores tanto extra-ovarianos como nutrição, idade e estresse da fêmea, como intra-ovarianos relacionados à comunicação intercelular, diâmetro oocitário além das condições do ambiente folicular, in vivo, ou do sistema de cultivo, in vitro (Krisher, 2013). Este contexto tem despertado o interesse da comunidade científica quanto a compreensão dos mecanismos bioquímicos e gênicos que regem a aquisição de competência oocitária assim como dos fatores extrínsecos e intrínsecos que interferem neste processo a fim de estabelecer marcadores de competência (Mermillod et al., 2008). Sendo assim, dada a relevância do tema, a presente revisão tem como intuito compilar e discutir de maneira sucinta os principais avanços científicos no que diz respeito à capacitação oocitária e fatores interferentes, tanto in vitro quanto in vivo.

\section{Qualidade Oocitária \\ Determinando o Potencial de Desenvolvimento de Oócito}

Em mamíferos, a oogênese inicia com a colonização da gônada primordial pelas células sexuais germinativas, também conhecidas como gonócitos, que, após intensas mitoses, se diferenciam em oócitos primários. Ainda durante a vida fetal, estes oócitos iniciam a meiose a qual é bloqueada no estágio diplóteno da prófase I, morfologicamente reconhecido pela vesícula germinativa (VG) e, assim, permanecem por meses ou décadas, dependendo da espécie animal. A partir da puberdade, a cada ciclo estral, um grupo de oócitos é recrutado e a divisão meiótica é retomada sob estímulo hormonal próximo a ovulação, com progressão até metáfase II (Mehlmann, 2005). No entanto, de todos os oócitos recrutados, apenas uma pequena proporção alcança o estágio ovulatório enquanto a grande maioria entra em atresia e degenera (Mermillod et al., 2008).

Este processo de segregação cromossômica caracteriza a maturação nuclear do oócito e envolve tanto modificações estruturais quanto recombinação genética, se completando apenas com a fertilização. A competência oocitária para suportar a embriogênese inicial depende, no entanto, não apenas dos eventos nucleares, mas também, da progressão sinérgica da maturação citoplasmática caracterizada pelo desenvolvimento e migração das organelas além do estoque de mRNAs e proteínas envolvidos na regulação de inúmeros processos celulares (Ferreira et al., 2008). Neste contexto, as células da granulosa também têm especial participação uma vez que, através da transferência de fatores de baixo peso molecular via junções GAP comunicantes (GJCs), regulam tanto o bloqueio quanto o reinício meiótico assim como as demais alterações a nível molecular e estrutural, por meio da ativação de segundo mensageiros e quinases, garantindo a adequada capacitação oocitária (Gilchrist et al., 2008).

A qualidade oocitária pode ser definida, portanto, como a competência do oócito em completar a maturação e suportar a fertilização e embriogênese inicial, seja in vivo ou in vitro, sendo resultante da interação de múltiplos eventos (Sirard et al, 2006). Segundo Krisher (2004), oócitos que não completam de maneira adequada as transformações pertinentes à maturação são considerados de baixa qualidade e resultarão em 
falhas durante a fertilização e embriogênese. O potencial de desenvolvimento dos complexos cumulus-oócitos (COCs), no entanto, está na dependência ainda de fatores intrínsecos e extrínsecos os quais envolvem a condição fisiológica, ambiental e até mesmo biotecnológica (Merton et al., 2003).

\section{Complexo Cumulus-oócito:}

\section{Importância da Interação Cumulus-Oócito para} Aquisição de Competência

As células do cumulus correspondem a um grupo de células da granulosa que durante a folículo-oogênese se diferencia e estabelece íntima comunicação com o oócito através das GJCs constituindo a unidade funcional designada COCs Esta interação é fundamental para coordenação da maturação nuclear e citoplasmática e consequente aquisição de competência oocitária (Gandolfi et al., 2005). As células da granulosa que constituem a parede do folículo, designadas células murais da granulosa ou células foliculares, também são imprescindíveis uma vez que, através da produção do fluido folicular, que consiste numa secreção ultrafiltrada do plasma sanguíneo composta por proteínas, hormônios, nutrientes e moléculas sinalizadoras, garantem a viabilidade e comunicação intrafolicular do oócito (Zamah et al., 2015).

Devido à maior proximidade com o oócito, as células do cumulus regulam, não somente, o processo de maturação, mas também, garantem ao oócito nutrição, através da transferência de metabólitos, íons e aminoácidos pelas GJCs, além de proteção e conexão com o meio externo. Adicionalmente, modulam os efeitos dos hormônios, facilitam a condução do oócito no oviduto após a ovulação, e direcionam os espermatozoides para a fertilização (Soom et al., 2002; Gandolfi et al., 2005; Gilchrist et al., 2008). Evidências demonstram ainda que o oócito não é uma estrutura passiva. Muito pelo contrário, através da síntese de fatores como o fator de crescimento e diferenciação 9 (GDF9) e a proteína morfogênica óssea 15 (BMP15), o oócito controla inúmeras funções das células da granulosa como proliferação, glicólise, secreção de moléculas sinalizadoras, expressão de receptores para LH, função esteroidogênica além da expansão do cumulus. Indiretamente, estes fatores de crescimento conferem ao oócito a habilidade de controlar seu próprio microambiente (Hussein et al., 2006; Russell et al., 2016).
Fatehi et al. (2002), em seus estudos com COCs de bovinos, observaram que a remoção das células do cumulus interferiu negativamente nas taxas de maturação, fertilização e desenvolvimento embrionário, confirmando a importância do suporte celular promovido por estas células através das GJCs. Além disso, oócitos desnudos apresentam maior incidência de anormalidades no ciclo celular, como progressão meiótica acelerada, inabilidade em manter o bloqueio em metáfase II e propensão a ativação espontânea (Combelles et al., 2002). A ausência dos fatores de crescimento sintetizados pelo oócito também afeta as células do cumulus que tendem à apoptose (Hussein et al., 2006). Deste modo, os métodos de recuperação dos COCs do ambiente folicular podem comprometer a qualidade oocitária, devido à remoção mecânica das células do cumulus (Rodríguez et al., 2006).

A interrupção da transferência de moléculas regulatórias decorrente da perda das GJCs pela expansão do cumulus, caracterizada pela deposição de uma matriz extracelular rica em ácido hialurônico, resulta ainda no reinício meiótico oocitário. In vivo, este processo é desencadeado progressivamente a partir do estímulo primário da onda pré-ovulatória de $\mathrm{LH}$ em COCs competentes. In vitro, em contrapartida, ocorre de maneira mais abrupta e acelerada em decorrência da remoção dos COCs do ambiente folicular, independente do seu estádio de desenvolvimento (Sasseville et al., 2009), contribuindo, assim, para assincronia da maturação nuclear e citoplasmática observada em oócitos cultivados (Combelles et al., 2002). Portanto, a presença das células do cumulus e o grau de expansão destas estão diretamente relacionados com o potencial de desenvolvimento oocitário.

\section{Fatores Interferentes}

\section{Aspectos Implicados na Aquisição de Competência Oocitária}

Devido as suas particularidades relacionadas ao ciclo celular, proporção núcleo:citoplasma e longevidade, o oócito consiste num tipo celular extremamente sensível à variações e, portanto, susceptível à fatores tanto extra-ovarianos relacionados à condição fisiológica da fêmea como nutrição, idade, metabolismo individual e estresse, quanto à fatores intra-ovarianos, os quais envolvem aspectos íntrínsecos dos COCs como comunicação intercelular e diâmetro oocitário, além das condições do ambiente folicular, in vivo, ou dos 
sistemas de cultivo e manipulação, in vitro (Mermillod et al., 2008; Krisher, 2013).

\section{Diâmetro Oocitário e Folicular}

In vivo, apenas folículos que estabeleceram dominância e alcançaram seu máximo desenvolvimento sofrem ação do LH culminando na ovulação de um oócito apto a fertilização (Pavlok et al., 1992). Segundo Hyttel et al. (1997), o diâmetro do folículo e do oócito está diretamente relacionado entre si e consiste, portanto, em um importante indicador de qualidade oocitária uma vez que reflete o grau de maturação citoplasmática caracterizado, principalmente, pelo estoque de proteínas e mRNA. Deste modo, de acordo com Marchal et al. (2002), quanto maior o diâmetro folicular e oocitário, maior competência para suportar os posteriores estádios do desenvolvimento. De fato, Lojkic et al. (2016) relataram clivagem mais acelerada e maior taxa de formação de blastocisto relacionadas ao maior diâmetro folicular. Ainda neste contexto, Feng et al. (2007) constataram que oócitos obtidos de folículos em início de atresia são mais competentes devido a exposição mais prolongada ao ambiente folicular e maturação avançada. No entanto, atresia em estágio avançado é prejudicial uma vez que resulta na degeneração celular.

Han et al. (2006), em contrapartida, constataram diferença significativa no potencial de desenvolvimento de COCs caprinos oriundos de folículos de mesmo diâmetro e com as características morfológicas similares quanto ao cumulus e ooplasma, o que sugere que a competência oocitária não é determinada apenas por um aspecto isolado, mas sim, por uma complexa interação de fatores intrínsecos e extrínsecos ao oócito. A própria composição do fluido folicular, em termos de ácidos graxos e glicose, reflete a constituição plasmática e metabolismo materno, exercendo influência direta sobre a viabilidade oocitária (Leroy et al., 2012). Neste contexto, pesquisas com proteômica têm sido realizadas visando buscar biomarcadores da qualidade oocitária no fluido folicular (Zamah et al., 2015).

\section{Idade Materna}

A possibilidade de recuperação de COCs desde animais pré-púberes até senis garante a otimização da fêmea doadora e obtenção de um número maior de descendentes. Evidências demonstram, no entanto, que existe uma relação direta entre a idade materna e o potencial de desenvolvimento oocitário, de modo que, oócitos oriundos de fêmeas senis são mais susceptíveis a aberrações cromossômicas, como aneuploidia, disfunção de organelas como retículo endoplasmático e mitocôndrias, e maior produção de radicais livres (Igarashi et al., 2015), enquanto oócitos de fêmeas pré-púberes tendem a apresentar maior incidência de alterações transcricionais e poliespermia (Paczkowski et al., 2011; Jiao et al., 2013). Deste modo, em ambos os casos, a competência oocitária para suportar a fertilização e os posteriores estádios do desenvolvimento é reduzida, resultando em baixas taxas de blastocisto, má formação e até mesmo falhas de implantação uterina.

A menor competência dos COCs obtidos de fêmeas pré-púberes tem sido atribuída a maturação citoplasmática insuficiente resultando em menor estoque de transcritos maternos e deficiência de moléculas regulatórias (Paczkowski et al., 2011). Yamamoto et al. (2010) também relatam perda precoce das junções comunicantes em complexos cumulus-oócitos de fêmeas senis resultando em reinício e progressão da meiose mais acelerados, o que pode predispor a assincronia da maturação oocitária observada in vitro. De acordo com Tamassia et al. (2003), existe, ainda, variação individual com relação a qualidade do oócito, demonstrando que o potencial de desenvolvimento oocitário é geneticamente controlado.

\section{Nutrição Materna}

O desbalanceamento nutricional materno pode resultar em alterações na composição química e hormonal do fluido folicular promovendo distúrbios metabólicos nos oócitos com redução significativa do potencial reprodutivo, sendo o excesso de nutrientes mais prejudicial que sua carência (Ashworth et al., 2009). De fato, Sohrabi et al. (2015), em seus estudos com ratos, constataram que dietas ricas em gordura, elevação dos níveis séricos de glicose, insulina e leptina assim como o excesso de peso afetam a expressão gênica, síntese proteica e atividade enzimática nas células da granulosa e no oócito resultando em falhas na maturação oocitária.

No entanto, em estudo realizado com novilhas, Adamiak et al. (2005) constataram que o efeito nutricional sobre a qualidade oocitária depende muito da condição de escore corporal (ECC) de modo que a alta taxa de ingestão em fêmeas gordas ou com ECC acima de 3,5, numa 
escala de 0 a 5 , resulta em hiperinsulinemia impactando de maneira negativa e cumulativa na viabilidade do oócito. Em contrapartida, a alta taxa de ingestão em fêmeas magras ou com baixo ECC $(2,0)$, melhora a fertilidade. Contudo, os mecanismos exatos implicados neste contexto ainda não estão completamente esclarecidos.

\section{Condições de Estresse}

O estresse consiste em outro importante fator que interfere negativamente na qualidade do oócito e fertilidade de diversas espécies animais inclusive no ser humano. Diversas situações podem representar estresse incluindo desde aspectos climáticos, nutricionais e de manejo aos quais as fêmeas são submetidas até variações locais do cultivo oocitário in vitro (Latham, 2016). Segundo Prasad et al. (2016), o aumento do nível circulante de cortisol é acompanhado por redução da concentração sérica de estradiol muito possivelmente devido a indução de estresse oxidativo nas células da granulosa que compõe o folículo e sustentam o desenvolvimento oocitário. De fato, Ozawa et al. (2005) relataram redução da atividade da aromatase e da expressão de receptores para $\mathrm{LH}$ nas células da granulosa justificando a redução no nível de estradiol em caprinos sob estresse térmico.

O metabolismo celular, naturalmente, gera radicais livres, também designados espécies reativas de oxigênio (ROS), como superóxido (O2-) e peróxido de hidrogênio (H2O2), os quais são degradados pelos sistemas enzimáticos e nãoenzimáticos de proteção antioxidante garantindo, deste modo, a homeostase (Prasad et al., 2016). Níveis fisiológicos de ROS são importantes na modulação de inúmeras funções das células da granulosa e do oócito. No entanto, qualquer desequilíbrio que resulte no acúmulo de ROS desencadeará modificações a nível transcricional e pós-traducional culminando, a longo prazo, em apoptose e deterioração celular. Neste contexto, o estresse crônico tem impacto mais severo sobre a viabilidade celular em detrimento ao estresse agudo (Latham, 2016; Prasad et al., 2016).

\section{Aspectos Biotecnológicos}

Biotecnologias reprodutivas envolvendo superestimulação ovariana, coleta de COCs imaturos e maturação in vitro têm impacto significativo sobre a qualidade oocitária podendo resultar em alterações a nível molecular e consequente redução do potencial de desenvolvimento e sobrevivência embrionária (Abd El-Aziz et al., 2016). Isso está relacionado, provavelmente, à exposição insuficiente dos COCs aos componentes foliculares, à inadequada maturação oocitária, à remoção mecânica das células do cumulus e às condições laboratoriais (Krisher, 2004; 2013). Logo, o cultivo in vitro deve refletir ao máximo o ambiente uterino de modo a possibilitar a adequada progressão da maturação além de prover nutrição e suporte para fertilização e embriogênese inicial (Abd El-Aziz et al., 2006).

Neste contexto, são preconizados meios definidos de maturação oocitária in vitro, como o TCM-199, suplementados com hormônios, fontes proteicas e energéticas (Farag et al., 2009). Dentre estes suplementos, o soro de origem fetal ou de fêmea em estro assim como o fluido folicular foram utilizados por muito tempo por conter componentes benéficos ao oócito, no entanto, sua composição indefinida e variável, além da sua origem animal, tem impulsionado a busca por substitutos como albumina sérica, álcool polivinílico, álcool pirrolidona, entre outros com igual função (Krisher et al., 2013).O fornecimento de substratos energéticos como glucose e piruvato também é imprescindível para o metabolismo oocitário uma vez que o processo da maturação despende muita energia (Xie et al., 2016). A adequada progressão da maturação oocitária e expansão do cumulus dependem ainda do estímulo de hormônios como LH, FSH e estradiol, os quais podem ser usados isolados ou combinados (Farag et al., 2009).

A tensão de oxigênio durante o cultivo in vitro também consiste em fator crucial para eficiência da PIV uma vez que, embora fundamental para sobrevivência celular, alta concentração deste gás favorecerá maior formação de ROS. Ainda existe discrepância entre autores com relação ao nível de oxigenação ideal para maturação oocitária in vitro, podendo variar de $5 \%$ a $20 \%$ de acordo com a espécie em questão e o meio utilizado (Hashimoto et al., 2000; Park et al., 2005). Uma alternativa para amenizar os efeitos do estresse oxidativo, manter a homeostase e incrementar a competência oocitária consiste ainda na utilização de antioxidantes como vitaminas e compostos tióis (Krisher et al., 2013).

O oócito não tem habilidade para regular o seu potencial hidrogeniônico $(\mathrm{pH})$ ficando na dependência das células do cumulus e da presença de soluções tampões no meio de cultivo. $\mathrm{O}$ ph interfere na atividade das organelas, em especial das mitocôndrias, assim como na formação do fuso 
meiótico (Krisher, 2013). Ainda neste contexto, dada a importância das células do cumulus, peculiaridades do método de coleta dos COCs por aspiração folicular, como calibre da agulha e pressão de aspiração também podem afetar de maneira significativa a qualidade oocitária por promover a remoção mecânica do cumulus. A eficiência de recuperação dos COCs, em termos de qualidade e quantidade, está na dependência ainda do operador da aspiração, diâmetro folicular e tratamento hormonal (Rodríguez et al. (2006).

No que diz respeito à superestimulação ovariana, esta possibilita que um número maior de folículos, que outrora entrariam em atresia, alcance o estádio ovulatório. Em contrapartida, se observa aumento da incidência de falhas na segregação cromossômica, alterações a nível transcricional e redução da viabilidade oocitária embrionária, podendo interferir até mesmo na implantação e sobrevivência do embrião no ambiente uterino. $\mathrm{O}$ efeito preciso dos hormônios exógenos sobre o oócito ainda não está completamente esclarecido (Cree al., 2015; Abd El-Aziz et al., 2016).

É importante ressaltar que a composição do meio de cultivo em termos de concentração e constituintes, nível ideal de oxigenação e ph estão ainda na dependência da espécie com a qual se trabalha. Além disso, materiais plásticos assim como a água ultra purificada utilizada no preparo do meio e o óleo mineral sob o qual os oócitos e embriões são cultivados podem promover toxicidade celular em nível variado (Krisher, 2013).

\section{Seleção de COCs Competentes}

\section{Aspectos que direcionam a escolha de COCs de boa qualidade}

Considerando os inúmeros fatores implicados na aquisição de competência oocitária, diferentes critérios considerados preditivos do potencial de desenvolvimento têm sido propostos para seleção de COCs competentes visando maior eficiência biotecnológica (Goovaerts et al., 2010). Dentre estes, a avaliação morfológica dos COCs, sob estereomicroscópio, com base nas características das células do cumulus e do ooplasma consiste no método de seleção usualmente preconizado na PIV dada sua facilidade de aplicação. Deste modo, COCs com várias camadas de células do cumulus compactas e ooplasma homogêneo são considerados de alta qualidade, enquanto que COCs com cumulus esparso, em menor quantidade ou expandido e ooplasma escuro e intensamente granulado são considerados de baixa qualidade, devendo ser descartados (Blondin e Sirard 1995).

O diâmetro folicular e oocitário assim como sinais de início de atresia caracterizada pela sutil expansão do cumulus e ligeira granulação do ooplasma também podem ser utilizados como indicador de competência. No entanto, por ser subjetiva, a avaliação morfológica é insuficiente para predizer de maneira fidedigna a qualidade dos COCs, uma vez que estes podem apresentar alterações, em termos metabólicos e moleculares, não identificados visualmente (Marchal et al., 2002; Feng et al., 2007).

A mensuração de parâmetros intrínsecos do oócito, como atividade mitocondrial, concentração intracelular de cálcio, atividade de fosfodiesterases e expressão de determinados genes também consiste num método alternativo para determinação da competência oocitária (Wang e Sun, 2007). Todavia, por ser invasivo e requerer a destruição dos COCs, tem aplicação limitada (Mermillod et al., 2008). Dada a íntima relação entre o oócito e as células do cumulus, a análise da viabilidade e metabolismo destas últimas assim como do fluido folicular através da mensuração de possíveis biomarcadores, como fatores de crescimento e proteínas específicas, consiste numa estratégia muito estudada. A dificuldade neste contexto seria a aspiração individual dos COCs sem contaminação sanguínea e com o mínimo de traumas, além, de envolver métodos de análise custosos (Leroy et al., 2012; Zamah et al., 2015).

A taxa de formação de blastocisto assim como o desenvolvimento e sobrevivência embrionária, estabelecimento e manutenção da gestação também refletem a qualidade do oócito, contudo, representam um critério de avaliação muito tardio (Mermillod et al., 2008). Do mesmo modo, a análise do perfil metabólico a partir do meio de cultivo oocitário e embrionário consiste em método preditivo não invasivo, mas, requer cultivo individual e fornece informações apenas após a execução da PIV (Goovaerts et al., 2010).

Neste contexto, uma alternativa adicional simples que auxilia na inferência do potencial de desenvolvimento sem danificar o oócito consiste na coloração com Azul de Cresil Brilhante (BCB). Este método se baseia na capacidade da enzima glucose-6-fosfato dehidrogenase (G6PDH), implicada na síntese de nucleotídeo e ácidos graxos, em metabolizar o corante. Sendo assim, COCs no início do desenvolvimento aparecem 
fracamente corados (BCB-) devido à maior atividade da G6PDH, enquanto que, com o avançar da maturação, a coloração se intensifica (BCB+) devido à redução da atividade enzimática. Evidências demonstram que $\mathrm{COCs} \mathrm{BCB}+$ resultam em melhores taxas de clivagem e blastocisto. Seu uso isolado, todavia, pode gerar resultados dúbios (Karami-Shabankareh et al., 2012).

\section{Considerações Finais}

Portanto, em se tratando de eficiência reprodutiva, tanto in vivo quanto nos sistemas de produção in vitro, a qualidade do oócito consiste num aspecto crucial uma vez que determina o potencial de desenvolvimento embrionário na dependência da interferência de inúmeros fatores extra e intra-ovarianos. Neste contexto, o aprimoramento do conhecimento é imprescindível a fim de possibilitar oócitos competentes e melhorias nas biotécnicas reprodutivas.

\section{Referências}

Abd El-Aziz, A.H.; Mahrous U.E.; Kamel, S.Z.; Sabek, A.A. Factors influencing in vitro production of bovine embryos: a review. Asian Journal of Animal and Veterinary Advances, 11: 737-756, 2016.

Adamiak, S.J.; Mackie, K.; Watt, R.G.; Webb, R.; Sinclair, K.D. Impact of nutrition on oocyte quality: cumulative effects of body composition and diet leading to hyperinsulinemia in cattle. Biology of Reproduction, 73:918-926, 2005.

Ashworth, C.J.; Toma, L.M.; Hunter, M.G. Nutritional effects on oocyte and embryo development in mammals: implications for reproductive efficiency and environmental sustainability. Philosophical Transactions of the Royal Society of London. Biological Sciences, 364(1534):3351-3361.

Blondin, P.; Sirard, M.A. Oocyte and follicular morphology as determining characteristics for developmental competence in bovine oocytes. Molecular Reproduction and Development, 41:54-62, 1995.

Choudhary, K.K.; Kavya, K.M.; Jerome, A.; Sharma, R.K. Advances in reproductive biotechnologies. Veterinary World, 9(4): 388-395, 2016.

Combelles, C.M.; Racowsky, C.; Albertini, D.F. Assessment of nuclear and cytoplasmic maturation in in-vitro matured human oocytes. Human Reproduction, 17:1006-1016, 2002.
Cree, L.M.; Hammaond, E.R.; Shelling, A.N.; Berg, M.C.; Peek, J.C.; Green, M. P. Maternal age and ovarian stimulation independently affect oocyte mtDNA copy number and cumulus cell gene expression in bovine clones. Human Reproduction, 30(6):1410 1420, 2015.

Farag, I.M.; Girgir, S.M.; Khalil, W.K.B.; Hassan, N.H.A.; Sakr, A.A.M.; AdbAllah, S.M.; Ali, N.I. Hormone and culture media effect on in vitro maturation of oocytes effect of hormones, culture media and oocyte quality on in vitro maturation of Egyptian sheep oocytes. Journal of Applied Biosciences, 24:1520-1534, 2009.

Fatehi, A.N.; Zeinstra, E.C.; Kooij, R.V.; Colenbrander, B.; Bevers, M.M. Effect of cumulus cell removal of in vitro matured bovine oocytes prior to in vitro fertilization on subsequent cleavage rate. Theriogenology, 57:1347-1355, 2002.

Ferreira, E.M.; Vireque, A.A; Adona, P.R.; Meirelles, F.V.; Ferriani, R.A.; Navarro, P.A.A.S. Cytoplasmic maturation of bovine oocytes: Structural and biochemical modifications and acquisition of developmental competence. Theriogenology, 71: 836-848, 2008.

Feng, W.G.; Sui, H.S.; Han, Z.B.; Chang, Z.L.; Zhoum P.; Liu, D.J.; Bao, S.; Tan, J.H. Effects of follicular atresia and size on the developmental competence of bovine oocytes: A study using the well-in-drop culture system. Theriogenology, 67:1339-1350, 2007.

Gandolfi, F.; Brevini, T.A.L.; Cillo, F.; Antonini, S. Cellular and molecular mechanisms regulating oocyte quality and the relevance for farm animal reproductive efficiency. Scientific and Technical Review of the Office International des Epizooties, 24(1): 413-423, 2005.

Gilchrist, R.B.; Lane, M.; Thompson, J.G. Oocytesecreted factors: regulators of cumulus cell function and oocyte quality. Human Reproduction Update, 14(2): 159-177, 2008.

Goovaerts, I.G.; Leroy, J.L.; Jorssen, E.P.; Bols, P.E. Noninvasive bovine oocyte quality assessment: possibilities of a single oocyte culture. Theriogenology, 74(9):1509-1520, 2010.

Han, D.; Lan, G.; Wu, Y.; Han, Z.; Wand, H.; Tan, J. Factors affecting the efficiency and reversibility of roscovitine (ros) block on the 
meiotic resumption of goat oocytes. Molecular Reproduction Development, 73:238-246, 2006.

Hashimoto, S.; Minami, N.; Takakura, R.; Yamada, M.; Imai, H.; Kashima, N. Low oxygen tension during in vitro maturation is beneficial for supporting the subsequent development of bovine cumulus-oocyte complexes. Molecular Reproduction Development, 57(4): 353-360, 2000.

Hussein, T.S.; Thompson, J.G.; Gilchrist, R.B. Oocyte-secreted factors enhance oocyte developmental competence, Developmental Biology, 296(2): 514-552, 2006.

Hyttel, P.; Fair, T.; Callesen H.; Greve, T. Oocyte growth, capacitation and final maturation in cattle. Theriogenology, 47:23-32, 1997.

Igarashi, H.; Takahashi, T.; Nagase, S. Oocyte aging underlies female reproductive aging: biological mechanisms and therapeutic strategies. Reproduction Medicine and Biology, 14(4):159-169, 2015.

Jiao, G.Z.; Cao, X.Y.; Cui, W.; Lian, H.Y.; Miao, Y.L.; Wu, X.F.; Han, D.; Tan, J.H. Developmental potential of prepubertal mouse oocytes is compromised due mainly to their impaired synthesis of glutathione. Plos One. 8(3): e58018, 2013.

Karami-Shabankareh, H.; Mirshamsi, S.M. Selection of developmentally competent sheep oocytes using the brilliant cresyl blue test and the relationship to follicle size and oocyte diameter. Small Ruminant Research, 105:244-249, 2012.

Krisher, R.L. The effect of oocyte quality on development. Journal of Animal Science, 82:E14-E23, 2004.

Krisher, R.L. In vivo and in vitro environmental effects on mammalian oocyte quality. Annual Review of Animal Biosciences, 1: 393-417, 2013.

Latham, K.E. Stress signaling in mammalian oocytes and embryos: a basis for intervention and improvement of outcomes. Cell and Tissue Research, 363(1):159-167, 2016.

Leroy, J.L.M.R.; Valckx, S.; Sturmey, R.; Bossaert, P.; Van Hoeck V.; Bols, P.E.J. Maternal metabolic health and oocyte quality: the role of the intrafollicular environment. Animal Reproduction, 9(4):777-788, 2012.

Lojkić, M.; Uvodić, S.; Getz, I.; Samardžija, M.; Aladrović, J.; Maćešić, N.; Karadjole, T.; Bačić, G.; Matković, M.; Benić, M. The influence of follicle size on the developmental kinetics of bovine embryos. Veterinarski Arski Arhiv, 86: 613-622, 2016.

Marchal, R.; Vigneron, C.; Perreau, C.; Bali-papp, A.; Mermillod, P. Effect of follicular size on meiotic and developmental competence of porcine oocytes. Theriogenology, 57:15231532, 2002.

Mehlmann, L.M. Stops and starts in mammalian oocytes: recent advances in understanding the regulation of meiotic arrest and oocyte maturation. Reproduction, 130: 791-799, 2005.

Mermillod, P.; Dalbie`s-Tran, R.; Uzbekova, S.; Thelie, A.; Traverso, J. M.; Perreau, C.; Papillier, P.; Monget, P. Factors affecting oocyte quality: who is driving the follicle? Reproduction in Domestic Animals, 43(2): 393-400, 2008.

Merton, J.S.; Roos, A.P.W.; Mullaart, E.; Ruigh, L.; Kaal, L.; Vos, P.L.A.M.; Dieleman, S.J. Factors affecting oocyte quality and quantity in commercial application of embryo technologies in the cattles breending industry. Theriogenology, 5: 651-574, 2003.

Ozawa, M.; Tabayashi, D.; Latief, T.A.; Shimizu, T.; Oshima, I.; Kanai, Y. Alterations in follicular dynamics and steroidogenic abilities induced by heat stress during follicular recruitment in goats. Reproduction, 129:621630, 2005.

Paczkowski, M.; Yuan, Y.; Fleming-Waddell J.; Bidwell, C.A.; Spurlock, D.; Krisher, R.L. Alterations in the transcriptome of porcine oocytes derived from prepubertal and cyclic females is associated with developmental potential. Journal of Animal Science, 89(11): 3561-3571, 2011.

Park, J.L; Hong, J.Y.; Yong, H.Y.; Hwang, W.S.; Lim, J.M.; Lee, E.S. High oxygen tension during in vitro oocyte maturation improves in vitro development of porcine oocytes after fertilization. Animal Reproduction Science, 87(1-2): 133-141, 2005.

Pavlok, A.; Lucas-Hahn, A.; Niemann, $H$. Fertilization and developmental competence of bovine oocytes derived from different categories of antral follicles. Molecular Reproduction and Development, 31:63-67, 1992.

Prasad, S.; Tiwari, M.; Pandey, A.N.; Shivastav, T.G.; Chaube, S.K. Impact of stress on oocyte 
quality and reproductive outcome. Journal of Biomedical Science, 23:36, 2016.

Rodríguez, C.; Anel, L.; Alvarez, M.; Anel, E.; Boixo, J.C.; Chamorro, C.A.; Paz, P. Ovum pick-up in sheep: a comparison between different aspiration devices for optimal oocyte retrieval. Reproduction in Domestic Animals, 41:106-113, 2006.

Russell, D.L.; Gilchrist, R.B.; Brown, H.M.; Thompson, J.G. Bidirectional communication between cumulus cells and the oocyte: Old hands and new players? Theriogenology, 86(1): 62-68, 2016.

Sasseville, M.; Gagnon, M.C.; Guillemette, C.; Sullivan, R.; Gilchrist, R.B.; Richard, F.J. Regulation of gap junctions in porcine cumulus-oocyte complexes: contributions of granulosa cell contact, gonadotropins, and lipid rafts. Molecular Endocrinology, 23(5): 700-710, 2009.

Sirard, M.A.; Richard, F.; Blondin, P.; Robert, C. Contribution of the oocyte to embryo quality. Theriogenology, 65: 126-136, 2006.

Sohrabi, M.; Roushandeh, A.M.; Alizadeh, Z.; Vahidinia, A.; Vahabian, M.; Hosseini, M. Effect of a high fat diet on ovary morphology, in vitro development, in vitro fertilisation rate and oocyte quality in mice. Singapore Medical Journal, 56(10):573-579, 2015.
Soom, A.V.; Tanghe, S.; Pauw, I. D.; Maes, D.; Kruif, A. Function of the cumulus oophorus before and during mammalian fertilization. Reproduction in Domestic Animals, 37:144151, 2002.

Tamassia, M.; Heyman, Y.; Lavergne, Y.; Richard, C.; Gelin, V.; Renard, J.P.; Chastant-Maillard, $\mathrm{S}$, Evidence of oocyte donor cow effect over oocyte production and embryo development in vitro. Reproduction, 126: 629-637, 2003.

Xie, H.L.; Wang, Y.B.; Jiao, G.Z.; Kong, D.L.; Li, Q.; Li, H.; Zhen, L.L.; Tan, J.H. Effects of glucose metabolism during in vitro maturation on cytoplasmic maturation of mouse oocytes. Scientific Reports, 6:20764, 2016.

Wang, Q.; Sun, Q.Y. Evaluation of oocyte quality: morphological, cellular and molecular predictors. Reproduction, Fertility, Development, 19:1-12, 2007.

Yamamoto, T.; Iwata, H.; Goto, H.; Shiratuki, S.; Tanaka, H.; Monji, Y.; Kuwayama, T. Effect of maternal age on the developmental competence and progression of nuclear maturation in bovine oocytes. Molecular Reproduction \& Development, 77: 595-604, 2010.

Zamah, A.M.; Hassis, M.E.; Albertolle, M.E.; Williams, K.E. Proteomic analysis of human follicular fluid from fertile women. Clinical Proteomics, 12(1): 2-12, 2015. 